\title{
Supportive neurodevelopmental evidence for ADHD as a developmental disorder
}

\author{
A. Panzer and M. Viljoen \\ Department of Physiology, University of Pretoria, PO Box 2034, Pretoria 0001, South Africa
}

\section{Summary}

A baby is dependent on its primary caregiver (hereafter referred to as 'mother') for its emotional regulation. The development of emotional self-regulation is dependent on the growth and myelinisation of connections between cortical (control) and limbic (emotion) structures in the infant brain. The subcortical sympathetic limbic system is dominant from birth, and it is only at 14-18 months of age that the parasympathetic cortical inhibitory part develops. The maturation of specifically the right orbitofrontal cortex, which dominates both the sympathetic and parasympathetic limbic systems, is essential for the regulation of emotion for the rest of an individual's life. Behavioral hyperactivity, impulsivity and inattention are considered normal for children in the early practising phase (10-14 months). This stage is characterised by sympathetic dominance stimulated by the ventral tegmental limbic circuit. We hypothesise that children with Attention Deficit Hyperactivity Disorder remain stuck in this phase, and accordingly do not enter the next stage of emotional development, i.e., the late practising period, in which the lateral tegmental limbic circuit, which stimulates the parasympathetic system develops. Parental reactions, which may contribute to this block in emotional development, include: largely ignoring their child, until the child does something the parent disapproves of, then scolding the child, without consoling the child again afterwards. This leads susceptible children to develop defensive hyperactivity and inattention in order to avoid a shame state they are unable to cope with. Implications for therapy are that caregivers should be taught firstly to give lots of positive attention to their child, and if necessary to scold, to console the child immediately afterwards. If this can be achieved consistently, the child will have the chance to develop their parasympathetic lateral limbic circuit, and eventually right orbitofrontal dominance over both limbic circuits, which translates into the ability to self-regulate their emotional states.

\section{Background}

Attention deficit hyperactivity disorder is a disorder, which is commonly diagnosed, yet poorly understood. Halasz and Vance [1] recently stated that Attention Deficit Hyperactivity Disorder (ADHD) may be seen as a developmental disorder, and cited the disagreement over its existence and use of diagnostic criteria, and the influence of insecure attachment to primary caregivers in the genesis of this disorder. We agree that ADHD is a developmental disorder, and have a theory on the neurodevelopment of ADHD, which may provide further supportive evidence.

\section{Normal brain development}

A human infant is born with a decidedly immature brain. Brain development is experience-expectant, that is, it is dependent on environmental stimulation for maturation. For a newborn, environmental stimuli are presented mainly through the primary caregiver (hereafter referred to as 'mother'). The baby is dependent on its mother, not just for its fluid and temperature homeostasis, but also for its emotional regulation. The mother is thus the 'external regulator' of the neurochemistry of the infant's developing 
brain [2]. The development of emotional self-regulation is dependent on the growth and myelinisation of connections between cortical (control) and limbic (emotion) structures in the infant brain [3]. Emotional expression is mediated by the balance between the energy-mobilising sympathetic system and the energy-conserving parasympathetic system of the autonomic nervous system. The subcortical sympathetic limbic system is dominant from birth, and it is only at 14-18 months of age that the parasympathetic cortical inhibitory part develops [4]. The maturation of specifically the right orbitofrontal cortex, which dominates both the sympathetic and parasympathetic limbic systems, is essential for the regulation of emotion for the rest of an individual's life [4] and [5].

The period most relevant to our theory regarding the development of ADHD is the practising period, as described by Mahler [6]. The practising period is subdivided into an early (from 10-12 to 14 months) and late (14-18 months) phase. These are discussed in the next two sections.

\section{The early practising period}

The early practising period begins when the infant starts to walk, normally from about 10 to 12 months, and ends at about 14 months [6]. Infants in the early practising period are characterised by their hyperactivity, elation and distractability, which is a reflection of the dominance of the ventral tegmental limbic circuit, which stimulates the sympathetic part of the autonomic nervous system [4] and [6].

The emotional availability of the mother is a crucial growth promoting aspect of early developmental experiences [7]. This means that the mother must be available and able to regulate her child's affect. The mother's socioaffective inputs, as provided in psychobiological face-to-face transactions, generates positive affect and activity of the infant [4] and [7]. These socioaffective inputs promote the experiencedependent growth of the ventral tegmental mesocortical A10 dopamine neurons into the right orbitofrontal cortex [4]. The early practising period is thus critical for the maturation of the ventral tegmental limbic circuit [4]. We hypothesise that children with ADHD remain stuck in this period, and do not progress to the next stage of emotional neurodevelopment (discussed in more detail later).

\section{The late practising period}

The late practising period ranges from about 14 to 18 months [6]. In this period, the mother's role changes from that of caregiver and playmate to that of socialiser and teacher of what constitutes acceptable behaviour and what not [4]. This braking action of the mother inhibits the sympathetic ventral limbic circuit, and encourages the extensive innervation of the right orbitofrontal cortex by the parasympathetic lateral tegmental noradrenergic limbic circuit from the lower brain stem medullary formation (A2 noradrenergic) [4]. When the happily playing sympathetic dominant child is suddenly confronted with a disapproving mother, the child is plunged into a parasympathetic dominant 'shame' state [8]. At this stage, children cannot yet autoregulate this shame state, and need consoling from their mother in order to be able to play again (i.e., regain sympathetic dominance). This soothing interaction is called 'interactive repair' [9]. In the short term, maternal emotional regulation leads to a happier child, while in the longer term, it leads to the child's internalisation of emotional self-regulation [4]. This latter ability is crucial for all the child's future relationships, including the ability to regulate their own children's emotional states [4]. Emotional self-regulation is dependent on right orbitofrontal dominance of both limbic circuits, in which dopamine innervates the deeper layers and noradrenaline innervates the more superficial layers, a pattern which is unique in the adult cortex [4] and [10]. 


\section{Neurodevelopment in children with ADHD}

A wide variety of factors are thought to play a role in the etiology of ADHD, including genetic transmission, toxins, socioeconomic status, food additives and imbalance of brain chemicals [11] and [12]. Nevertheless, both the quality of parenting and the nature of the home environment are implicated strongly in most cases [12]. In ADHD the characteristic phenomena of overactivity, impulsivity and distractibility are usually attributed to a primary deficit in the development of cognitive processes involved in the inhibition of inappropriate responses [13]. This is compatible with our theory regarding the neurodevelopment of ADHD, which is based on the socioaffective interactions of the mother with her 14-18-month-old child. We hypothesise that the majority of experiences many of these children have with their mothers are bifold. Either, the mother largely ignores her child and the child carries on in a sympathetic state, without learning to respond to social cues (i.e., not being socialised). This is similar to the avoidant attachment described by Ainsworth and Witting [14]. When the mother does interact with the child, it is by scolding the child [15], who is then overwhelmed by negative emotions and frozen in a parasympathetic shame state. These mothers do not consistently console the child, and the children learn to fear these parasympathetic dominant shame states, because they are unable to autoregulate them [4]. Children are thus left to their own resources, and we hypothesize that this would mean that they would continue with the sympathetic dominant state, characterised by behavioural hyperactivity and inattention. They would learn to ignore their mothers as far as possible, avoiding eye contact, in fear of the parasympathetic state the mother can induce by scolding or humiliating the child. This would lead to impaired development of the orbitofrontal cortex and deficiencies in self-regulation, as have been demonstrated in children with ADHD [16], [17], [18] and [19]. Furthermore, abnormalities in dopamine transmission and adrenaline excretion have been shown in children with ADHD, and are related to inattention [20] and [21]. However, the exact abnormalities of the brain, as well as the autonomic system, are still controversial [22], [23], [24] and [25].

We thus hypothesise that ADHD is a developmental disorder in which children remain stuck in the early practising period developmental phase, which is normal for 10-14-month-old infants, and is characterised by hyperactivity, impulsivity and inattention.

\section{Therapeutic implications}

We agree that medication should be used as a last resort, and that family therapy could be very successful [1] and [11]. In particular, parents should learn firstly to give lots of positive attention to their child, and if necessary to scold, to console the child immediately afterwards. If this can be achieved consistently, the child will have the chance to develop their parasympathetic lateral limbic circuit, and eventually right orbitofrontal dominance over both limbic circuits, which translates into the ability to self-regulate their emotional states. This will obviate the need for children to develop defensive hyperactivity and inattention in order to avoid a shame state they are unable to cope with. 


\section{References}

[1] G. Halasz and A.L.A. Vance, Attention deficit hyperactivity disorder in children: moving forward with divergent perspectives, MJA 177 (2002) (10), pp. 554-557.

[2] M. Hofer, The mother as hidden regulator. In: R Plutchnik and H Kellerman, Editors, Emotion: theory, research and experience, Academic Press, New York (1983), pp. 199-219.

[3] H.C. Kinney, B.A. Brody and A.S. Kloman et al., Sequence of central nervous system myelination in human infancy, J Neuropath Exp Neurol 47 (1988), pp. 217-234

[4] A.N. Schore, Affect regulation and the origin of the self, Lawrence Erlbaum Associates, Hillsdale (1994).

[5] R. Joseph, The right brain and the unconscious: discovering the stranger within, Plenum Press, New York (1992).

[6] M. Mahler, F. Pine and A. Bergman, The psychological birth of the human infant, Basic Books, New York (1975).

[7] C. Trevarthen In: C. Trevarthen, Editor, Brain circuits and functions of the mind, Cambridge University Press, Cambridge (1990), pp. 334-363.

[8] G. Kaufman, The psychology of shame, Springer, New York (1989).

[9] E.Z. Tronick and J.F. Cohn, Infant-mother face-to-face interaction, Child Dev 60 (1989), pp. 85-92.

[10] D.A. Lewis, S.L. Foote and M. Goldstein et al., The dopaminergic innervation of monkey prefrontal cortex: a tyrosine hydroxylase immuno-histochemical study, Brain Res 449 (1988), pp. 225243

[11] R.D. Ladnier and A.E. Massanari, Treating ADHD as attachment deficit hyperactivity disorder. In: T.M. Levy, Editor, Handbook of attachment interventions, Academic Press, San Diego (2000), pp. 2765.

[12] M.T. Greenberg, M. DeKlyen, M.L. Speltz and M.C. Endriga, The role of attachment processes in externalising psychopathologies in young children. In: L. Atkinson and K.J. Zucker, Editors, Attachment and psychopathology, Guilford Press, New York (1997), pp. 196-222.

[13] C. Hollis and E. Taylor, Schizophrenia: a critique from the developmental psychopathology perspective. In: M.S. Keshavan and R.M. Murray, Editors, Neurodevelopment and adult psychopathology, Cambridge University Press, Cambridge (1997), pp. 224-232.

[14] M.D.S. Ainsworth and B.A. Wittig, Attachment and the exploratory behavior of one year olds in a strange situation. In: B.M. Foss, Editor, Determinants of infant behavior vol. 4, Methuen, London (1969), pp. 113-136.

[15] C. Magai, Affect, imagery and attachment: working models of interpersonal and the socialisation of emotion. In: J. Cassidy and P.R. Shaver, Editors, Handbook of attachment: theory, research and clinical applications, The Guilford Press, New York (1999), pp. 796-797. 
[16] S.H. Mostofsky, K.L. Cooper and W.R. Kates et al., Smaller prefrontal and premotor volumes in boys with attention-deficit/hyperactivity disorder, Biol Psychiat 52 (2002) (8), pp. 785-794.

[17] S. Itami and H. Uno, Orbitofrontal cortex dysfunction in attention-deficit hyperactivity disorder revealed by reversal and extinction tasks, Neuroreport 13 (2002) (18), pp. 2453-2457

[18] S. Sandberg, Hyperkinetic or attention deficit disorder, Brit J Psychiat 169 (1996) (1), pp. 10-17.

[19] G.C. Kaya, A. Pekcanlar and R. Bekis et al., Technetium-99m HMPAO brain SPECT in children with attention deficit hyperactivity disorder, Ann Nucl Med 16 (2002) (8), pp. 527-531.

[20] P. Rosa Neto, H. Lou and P. Cumming et al., Methylphenidate-evoked potentiation of extracellular dopamine in the brain of adolescents with premature birth: correlation with attentional deficit, Ann N Y Acad Sci 965 (2002), pp. 434-439.

[21] G.M. Anderson, M.A. Dover and B.P. Yang et al., Adrenomedullary function during cognitive testing in attention-deficit/hyperactivity disorder, J Am Acad Child Adolesc Psychiat 39 (2000) (5), pp. 635-643

[22] N.L. Girardi, S.E. Shaywitz and B.A. Shaywitz et al., Blunted catecholamine responses after glucose ingestion in children with attention deficit disorder, Pediatr Res 38 (1995) (4), pp. 539-542. [23] E. Mikkelsen, C.R. Lake and G.L. Brown et al., The hyperactive child syndrome: peripheral sympathetic nervous system function and the effect of d-amphetamine, Psychiatry Res 4 (1981) (2), pp. 157-169

[24] B. Hesslinger, L. Tebartz van Elst and T. Thiel et al., Frontoorbital volume reductions in adult patients with attention deficit hyperactivity disorder, Neurosci Lett 328 (2002) (3), pp. 319-321.

[25] E.J. Sonuga-Barke, Psychological heterogeneity in AD/HD - a dual pathway model of behaviour and cognition, Behav Brain Res 130 (2002) (1-2), pp. 29-36 